\title{
Mathematical modelling for antibiotic resistance control policy: do we know enough?
}

\author{
Gwenan M. Knight ${ }^{*}$ (D), Nicholas G. Davies ${ }^{1}$, Caroline Colijn ${ }^{2+}$, Francesc Coll ${ }^{3 \dagger}$, Tjibbe Donker $^{4 \dagger}$, Danna R. Gifford ${ }^{5 \dagger}$, \\ Rebecca E. Glover ${ }^{6+}$, Mark Jit ${ }^{1+}$, Elizabeth Klemm ${ }^{7 \dagger}$, Sonja Lehtinen ${ }^{8 \dagger}$, Jodi A. Lindsay ${ }^{9 \dagger}$, Marc Lipsitch ${ }^{10 \dagger}$, \\ Martin J. Llewelyn ${ }^{11 \dagger}$, Ana L. P. Mateus ${ }^{12 \dagger}$, Julie V. Robotham ${ }^{13 \dagger}$, Mike Sharland ${ }^{14 \dagger}$, Dov Stekel ${ }^{15 \dagger}$, \\ Laith Yakob ${ }^{16 \dagger}$ and Katherine E. Atkins ${ }^{1,17 \dagger}$
}

\begin{abstract}
Background: Antibiotics remain the cornerstone of modern medicine. Yet there exists an inherent dilemma in their use: we are able to prevent harm by administering antibiotic treatment as necessary to both humans and animals, but we must be mindful of limiting the spread of resistance and safeguarding the efficacy of antibiotics for current and future generations. Policies that strike the right balance must be informed by a transparent rationale that relies on a robust evidence base.

Main text: One way to generate the evidence base needed to inform policies for managing antibiotic resistance is by using mathematical models. These models can distil the key drivers of the dynamics of resistance transmission from complex infection and evolutionary processes, as well as predict likely responses to policy change in silico. Here, we ask whether we know enough about antibiotic resistance for mathematical modelling to robustly and effectively inform policy. We consider in turn the challenges associated with capturing antibiotic resistance evolution using mathematical models, and with translating mathematical modelling evidence into policy.
\end{abstract}

Conclusions: We suggest that in spite of promising advances, we lack a complete understanding of key principles. From this we advocate for priority areas of future empirical and theoretical research.

Keywords: Dynamic modelling, Antibiotic resistance (ABR), Antimicrobial resistance (AMR), Decision-making

\section{Background}

Mathematical modelling is a tool that allows us to integrate our mechanistic understanding of biological processes-such as the spread of antibiotic resistance (ABR) - in a precise and logical structure. A correctlyspecified model can not only reproduce the empirical patterns that we observe, but also enable us to predict how changing conditions may impact upon real-world

\footnotetext{
* Correspondence: gwen.knight@lshtm.ac.uk

Caroline Colijn, Francesc Coll, Tjibbe Donker, Danna R. Gifford, Rebecca E. Glover, Mark Jit, Elizabeth Klemm, Sonja Lehtinen, Jodi A. Lindsay, Marc Lipsitch, Martin J. Llewelyn, Ana L. P. Mateus, Julie V. Robotham, Mike Sharland and Dov Stekel are speakers at the RESIST workshop.

'Laith Yakob and Katherine E. Atkins contributed equally to this work

'Department of Infectious Disease Epidemiology, Faculty of Epidemiology and Population Health, London School of Hygiene and Tropical Medicine (LSHTM), London, UK

Full list of author information is available at the end of the article
}

outcomes. Since ABR is a priority issue for global health, policymakers are increasingly concerned about how best to manage the spread of ABR, and are engaged in designing new guidelines and policies for doing so. Mathematical modelling has the potential to help inform these policies because it can quickly and inexpensively predict the outcomes of different actions, including inaction. Here we discuss some of the progress that has been made in using modelling to shape policy, highlighting the challenges facing the field and identifying future research priorities. We do this by first considering how far mathematical models have come in capturing antibiotic resistance evolution and discussing the remaining challenges. Then we evaluate how these models have been successful in guiding decision-making and the questions that remain. 


\section{Main text}

\section{Capturing antibiotic resistance evolution with} mathematical models

Before a mathematical model is deployed in decisionmaking, it must first convince us of its explanatory capabilities. In other words, before a model can be used as a reliable guide for policy, it must be able to recapitulate the empirically-observed prevalence of resistance - typically reported as either the number of cases of resistant infections or the proportion of bacterial isolates exhibiting resistance - at the appropriate local, regional, national or international level. This is not a simple task. Fully capturing these observations 'from the ground up' requires understanding: (i) how bacteria acquire resistance, whether by horizontal transfer of resistance genes or de novo mutation [1]; (ii) how these resistant cells proliferate, both within and between hosts; (iii) which forces, including antibiotic exposure, select for the transmission of resistant over non-resistant strains across diverse environments; (iv) how the circulation of resistant strains translates to reported numbers of infections or carriage episodes of resistant strains in different settings, for each "bug-drug" combination; and (v) how diagnostic, sampling, culture and typing methods affect our data on $\mathrm{ABR}$ incidence and prevalence.

\section{What we know}

Selection for and against antibiotic resistance The basis for the dynamics of antibiotic resistance is Darwinian evolution. The presence of an antibiotic selects for a higher frequency of organisms resistant to that antibiotic, because resistance to treatment confers those strains a benefit over susceptible strains [1]. Conversely, many models have assumed that resistance genes impose costs for the bacteria that carry them, resulting in resistant bacteria having lower fitness in the absence of antibiotics [2] — an assumption which is generally, but not universally, borne out by observation $[3,4]$. Accordingly, the strength of selection for resistance depends upon the balance between the benefits and costs of resistance. A corollary of assigning a cost to resistance is the 'time-reversibility' of evolution - that is, if antibiotic use is removed, resistance is counterselected and should equilibrate to the same frequency as before the introduction of the antibiotic [5]. Further, the between-host transmission of resistant bacterial strains, as opposed to de novo mutation or horizontal acquisition of resistance genes by bacteria, is generally assumed to be an important driver in the maintenance of antibiotic resistance [6]. These principles are naturally articulated within mathematical models that capture the dynamical processes of transmission, colonization, and treatment.

Competition (likely) exists between resistant and sensitive strains While some models of ABR account only for the transmission of resistant strains, there is growing recognition that tracking the dynamics of sensitive strains is important as well [6], especially if these strains are competing for limited resources: a finite niche within an individual person, and a finite number of people to colonise. These competitive dynamics substantially impact resistance evolution in both empirical studies [7-9] and theoretical mathematical models [10-12]. These modelling studies emphasize that competition between resistant and sensitive strains can occur both at the within- and the between-host level, and the relative importance of competition at these two levels can drive resistance evolution in opposing directions [13]. Competition also occurs between commensal and pathogenic bacteria occupying the same niche, with some unculturable competitors that are also affected by antibiotic exposure; this has only recently come to light with the advent of rapid affordable deep sequencing technology and associated analysis [14]. Further theoretical work and empirical investigation will permit a more precise characterisation of the competitive dynamics between resistant and sensitive strains, allowing us to establish ecologically sound principles for modelling competition both within and between hosts.

Transmission networks and heterogeneity of exposure to antibiotics Modelling is beginning to help us understand the geographic networks of ABR transmission $[15,16]$ in hospitals, communities, agricultural settings, and the environment. Paired with analysis of UK patient movement data, modelling has revealed the importance of locally circulating ABR [17]. Local outbreaks in ABR hotspots such as hospitals and long-term care facilities, which feature high antibiotic use and, often, immunocompromised patients, are generally better documented than broader patterns of community acquisition. Where detailed patient data do exist - often in the intensive care setting - stochastic mathematical models are now being used to assess the extent of transmission attributable directly to patients, healthcare workers or indirectly to the facility's environment [18]. Combining mathematical and phylodynamic modelling in the advent of cheaper sequence data is likely to present new opportunities to further understand the sources of health care-acquired resistant infections [19]; a better understanding of the role that non-patients and healthcare workers can play in resistance outbreaks may follow. Further, modelling has also been used to suggest that a greater proportion of antibiotic resistant bacteria is acquired in the community than in the hospital setting $[20,21]$, and hence that antibiotic stewardship efforts should include the community. While the importance of agricultural antibiotic use for human health is debated, modelling results have suggested that curtailing antibiotic growth promotion in livestock will be of less benefit than reducing 
animal-to-human transmission [22, 23]. Sequence data is likely to further our understanding of transmission from agricultural sources [24, 25]. All told, mathematical modelling is helping us to understand how resistance spreads in specific settings and within specific groups.

\section{Challenges remaining}

Lack of precise understanding of selection pressure Beyond the empirically well-supported hypothesis that greater antibiotic use by individuals in a population selects for a higher frequency of resistance among bacteria circulating in that population [26, 27], we have not yet convincingly identified the major drivers of the spread of resistance at the population level. One difficulty lies in explaining what maintains coexistence between resistant and non-resistant strains over long periods of time, when simple models predict that, depending upon the average antibiotic consumption rate in a population, either resistant or sensitive strains should competitively exclude the other [6,28]. A number of recent studies have proposed potentially complementary mechanisms - e.g. balancing selection caused by within-host competition [10, 28], variable selection for resistance over heterogeneous genetic backgrounds $[29,30]$, or population heterogeneity in treatment rates $[28,31]$ - which may each be capable of explaining this empirically-observed coexistence [13]. The relative importance of these and other mechanisms will vary depending upon the pathogen and setting, but remains to be identified for any one case.

A further difficulty in characterizing selection pressures for resistance is that a substantial proportion of variation in resistance to specific antibiotics between populations is not explained by variation in the consumption of those antibiotics: identifying interactions between co-selection of resistance determinants $[29,30]$, bystander selection [31], and other forces selecting for resistance is crucial for a complete understanding of resistance evolution. In principle, model calibration to empirical data could help to choose between alternative mechanisms. There is no shortage of hypotheses for what may contribute to the spread of resistance; what is needed is convincing empirical evidence to identify the most important forces shaping resistance evolution. Finally, on top of these uncertainties, mathematical models overlay a suite of additional and much less understood assumptions - the within-host dynamics of the bacteria within the microbiome, the social mixing patterns of individuals and the existence and strength of coselection. It is precisely the interplay between all these factors that drastically changes what the models actually predict $[11,13]$.

Setting-specific model calibration and data availability Recent work has begun to calibrate models to empirical data on the relationship between antibiotic consumption and resistance $[10,13]$, with the aid of databases linking antibiotic use and resistance at a country or state level, such as the European Centre for Disease Prevention and Control's European Antimicrobial Resistance Surveillance Network (ECDC EARS-Net) [32], the Center for Disease Dynamics, Economics \& Policy ResistanceMap [33], and the World Health Organization's Global Antimicrobial Resistance Surveillance System (GLASS) [34], as well as a host of national surveillance systems. However, these data appear insufficient to distinguish the mechanisms that govern the selection pressure that underpins the dynamics of resistant strains [13]. While, to date, this calibration has only been attempted in the context of a small subset of bacteria-treatment combinations, it is likely that these general limitations will extend to the wider group of pathogens. To distinguish the mechanisms or set of mechanisms generating the resistance dynamics we observe will require investigation of within-host strain diversity, strain epidemiology, and the demography and geography underlying transmission. It will also require consideration of data and properties of a more diverse set of potentially-pathogenic bacteria, as well as commensal and environmental bacteria, than the commonly used example of Streptococcus pneumoniae [10, 13, 28, 29, 35].

Transmission networks and heterogeneity of exposure to antibiotics Despite the advances in using mathematical models to disentangle the role of different groups of hosts in the transmission of resistance, elucidating the connections between, and the relative importance of, the heterogeneous environments in which resistance evolves remains a key problem. Both hotspots of ABR acquisition (which could be related to geographical areas/types of food-production systems/healthcare settings) and the most relevant pathways for exposure are unclear. For modelling to inform where to direct interventions, it must span these diverse environments. In doing so, it has the potential to help resolve some of the most contentious debates in ABR policy, such as the relative importance of agricultural, environmental, community and health-care reservoirs as sources of resistant infections.

Difficulty in quantifying fitness costs Open questions remain regarding how to quantify the fitness costs associated with resistance. How large are the costs? Do they manifest as reductions in within-host growth, betweenhost transmissibility, infectivity, or all three? Mathematical modelling can, in principle, be used to estimate the magnitude of fitness costs associated with resistance directly from epidemiological data [10, 13, 36-39]. However, fitness is a highly location-, time- and strain-specific characteristic $[40,41]$; care must be taken not to overgeneralise. As mathematical modelling predictions depend crucially 
on competitive strain dynamics, which in turn depend on resistance cost, the unknown effect of a combination of synergistic or antagonistic interactions [42] make model predictions highly uncertain. Moreover, although it is implicitly assumed by mathematical models, there is no overwhelming evidence to suggest that costs to resistance genes are unavoidable, whether truly cost-free resistance mutations will eventually arise, or whether backmutations towards lesser resistance would spread under a reduction of antibiotics.

Translating mathematical modelling evidence into policy Mathematical modelling has the potential to test policy interventions in silico, and hence to help us both understand the relevant components in complex systems and assess their relative impact and potential cost-effectiveness both as standalone policies [43-45] and as elements of combination ("bundled") policies [46]. This approach can then be used to predict the impact of updating the interventions or extending them long term.

\section{What we know}

The usefulness of mathematical models for health policy decision-making Although a comprehensive overview of the use of mathematical models in health policy [47] cannot be given here, it is clear that infectious disease models currently provide crucial evidence for public health decision making in many areas. A prime example is the use of mathematical models to support vaccination recommendations by National Immunization Technical Advisory Groups (NITAGs), such as the UK's Joint Committee on Vaccination and Immunisation (JCVI) [48]. In this and other well-established areas of health policy, predictions from mathematical models are translated into health economic terms by expressing health burdens in standardised units, e.g. quality-adjusted life years (QALYs) or disability-adjusted life years (DALYs). This allows the efficiency and affordability of alternative interventions to be assessed and compared in terms of the monetary cost per QALY gained or DALY averted. Although at present this economic framework is not widely used for questions relating to the control of resistant infections, recent estimates of resistance-attributable standardised health burdens $[49,50]$ are beginning to make this possible [51].

\section{Challenges remaining}

Lack of validated models As we have discussed above, the widespread use of dynamic modelling is lacking for many current $A B R$ control policies due to the challenges we face in understanding and quantifying $A B R$ transmission [52]. This can mean that we lack a framework for assessing interventions that are rolled out. For example, in the UK, the impact of a recent policy change from broad to narrow spectrum antibiotic usage, with a particular focus on reducing the rate of $C$. difficile infection [53], was not supported with predictive modelling, potentially hampering our ability to optimally assess this intervention in a timely manner. In general, a key function of dynamic models is to predict the time scale on which changes are expected to occur following interventions, and in general this has not been done systematically despite some efforts $[13,54,55]$. As a result, ABR modelling is underdeveloped relative to other areas of infectious disease modelling that support decision-making, such as vaccine policy where model calibration is a key requirement for a model to be fit for purpose $[56,57]$.

Questions of outcomes In managing the challenge of antibiotic resistance, our goal is not to reduce resistance per se, but to mitigate the health burdens that are caused by resistance. In other words, resistance is only a problem insofar as it leads to worse health outcomes. But how to calculate the attributable health burdens of resistance is an active area of research, and accordingly this remains a barrier to developing informed policy. Rather like climate change, policies must be enacted now to have an impact in the long term [58], but the potential long-term benefits of avoiding resistance must be balanced against the low cost, convenience, and lifesaving potential of antibiotics. Hence, a key area for modelling is the burden of current and future $A B R$, in terms of morbidity, mortality and economic impact: widelycited cited projections use have been produced for worldwide ABR burden by 2050 [59], while current and future burden has been estimated in more rigorous frameworks for European countries [50, 60], but better data and methods of attribution are needed to inform parameters such as attributable mortality $[49,61]$. Moreover, predictions of future burdens should be tied where possible to a mechanistic understanding of how resistant infection incidence is likely to evolve over time, as described in the prior section.

A complication of quantifying the attributable burden of resistance lies in identifying the counterfactual to a resistant infection: that is, whether calculating the health burden of resistance requires comparing a resistant infection to a susceptible infection, or to no infection at all. This counterfactual would not be the same for all pathogens and settings [62]. The incidence of the syndrome will also vary: for total burden it is the combination of prevalence of resistance with incidence of syndrome that matters, and these in turn may be affected by rates of antibiotic use and/or prevalence of resistance [63]. Reducing this complexity down to an index that can be easily communicated can give insight into how resistance levels are changing in time and 
space [64]. These estimates are important not only for policy makers, but also for properly incentivizing the development of new antibiotics.

The case of antibiotic stewardship A key intervention is antibiotic stewardship: preserving the efficacy of antibiotics by limiting their unnecessary use, optimising dosages and durations of treatment, and using drugs or combinations of drugs that limit selection for resistance. A major impediment to effective stewardship is that we do not know exactly what features of antibiotic use drug, dosage, length versus frequency of treatment episodes - are most important for promoting resistance, and yet these factors may have a significant impact upon resistance evolution (e.g. [35, 65]) and could help to explain the variation in resistance between settings that is not explained by the volume of antibiotic consumption alone. This may be due to the complexity of determining how to measure resistance and antibiotic consumption, how to weigh up the importance of antibiotic use across different populations (e.g. humans versus livestock), and what constitutes "appropriate" treatment [66].

In some settings, policies reducing antibiotic use-either overall or within specifically targeted classes- have been associated with reductions in resistance [67-71]; however, these conclusions have not been universal (e.g. $[72,73])$. Results vary due to key unknowns: notably, whether reduced antibiotic use will always reduce resistance, at what rate increased use will increase resistance, whether a given population is at equilibrium resistance prevalence and how fast these equilibria are reached. With this level of uncertainty, mechanistic models are often unable to robustly capture the dynamics and instead statistical trend prediction or machine learning has been employed. For example, in analysing trends for 72 pathogen-antibiotic combinations across the United States, statistical modelling has recently suggested that broadly-distributed, low-intensity use was more strongly associated with resistance levels than repeated use of antibiotics [74]. Since repeated use might represent the 'low-hanging fruit' of antibiotic stewardship efforts, this finding highlights a potential policy challenge.

One area where discussion of ABR policy has been most led by mathematical models is the long-standing debate over whether rotating antibiotics (that is cycling the use of a single antibiotic class within a single population) or using different combinations (mixing antibiotic classes within one population or combining antibiotics classes within individual patients) better prevents resistance acquisition (see [6] for a wider discussion). Diverse predictions provide insight into underlying process, but prevent universal conclusions from being drawn and modelling may be best viewed as complementary to clinical trials [75]. This highlights how far we have to go to understand the selection and transmission of resistance under antibiotic treatment.

The case of vaccination Vaccination has been proposed as a means of mitigating the burden of resistant infections [59]. Bacterial vaccines can be used to prevent infections that may otherwise require treatment with antibiotics, while viral vaccines can prevent diseases such as influenza which are often treated inappropriately with antibiotics. Mathematical frameworks have been developed for modelling the broader reduction in prevalence of infection due to vaccines $[59,76]$, as well as for estimating the impact of viral vaccines on antibiotic use and resistance [51]. However, the long-term impact of bacterial vaccination on the evolution of antibiotic resistance is complex (reviewed in [45]), and uncertainties over what drives resistance evolution lead to varying predictions concerning whether vaccination inhibits or promotes the long-term evolution of antibiotic resistance, where the nature of competition between resistant and sensitive strains has been identified as crucial for determining the impact of bacterial vaccination on resistance $[13,77]$.

The case of diagnostic tests The promise of rapid diagnostic tests - or substitutes such as machine-learningguided clinical histories [78] - is the potential to alleviate some of the uncertainties surrounding which antibiotics should be prescribed for a suspected bacterial infection. Nonetheless, the evidence of clinical impact on antibiotic use is sparse [78-80] and few studies have investigated the impact on antibiotic use or resistance [52]. In this situation, modellers must work closely with microbiologists and clinicians to develop tools that correctly capture what is being empirically measured as well as guiding surveillance system design; only then can models precisely determine the relative impact of interventions. It will be important to distinguish the short-term benefits of optimizing treatment [78] from the longer-term effects of more appropriate treatment on the evolution of resistance [81].

The case of clinical trials A significant barrier to determine competing risks of policy interventions is the lack of standardisation of resistance outcomes in current clinical trials. There is a limited number of strategic trials comparing alternative antibiotic regimens, but the majority either do not measure ABR outcomes at all, or compare different types of clinical samples, taken at different times, with widely varying phenotypic and genotypic methods. This makes comparison between studies very difficult and prevents the assessment of optimal outcomes from an "ABR perspective". There is an urgent need to provide some harmonisation and guidance on assessment of resistance outcomes - including some early form of standardisation of units of resistance at an 
individual and population level [64]. Major policy interventions under consideration, for example, mass drug treatment with azithromycin [82], will include formal drug toxicity and clinical cost benefit assessments, but cannot currently include any formal assessment of adverse effects on drug resistance in the population as there is no standard methodology to use. This has the effect of downgrading potential ABR adverse outcomes, with policy decisions driven by cost/toxicity factors that can be formally measured. This inevitably limits the modelling support that can be done to aid intervention design for ABR control.

Prioritizing resources Determining where to target policies - for example, towards the agricultural community or at interventions such as improved sanitation - is hampered by a lack of quantification of the source and drivers of ABR [83]. Building modelling into established protocols for decision making, such as is done for vaccines in the UK [48] and formally assessing interventions as they are rolled out would improve decision making. In particular, models that determine the differential impact of interventions by geographical setting could be used to inform the development of national action plans [84].

Surveillance of trends Fundamentally, modelling for policy requires some assessment of trend: to improve modelling requires better granular surveillance data on trends in ABR in different environments (e.g. [85]) as well as a better understanding of the relationships driving these trends. Reduction in ABR must also be consistent with policy aims-such as reducing overall infection prevalence or mortality-which may require increasing the use of antibiotics. Modellers must be sensitive not only to ABR dynamics, but the context within which a given policy sits.

\section{Conclusions}

Mathematical models are needed to make good decisions about how to manage ABR, because they make understanding the complexities of resistance evolution more manageable. Therefore, the mechanistic framework of mathematical models provides a valuable opportunity to both quantify ABR transmission and understand how to optimise usage of antibiotics and other interventions. Mechanistic models implicitly capture aspects of antibiotic resistance that we find more intuitive, such as the selection of antibiotic resistance in the presence of antibiotics and the existence of fitness costs of resistance. Accordingly, mathematical models can also help us to formulate novel ways of managing resistance.

However, the current state of mathematical modelling of ABR has both conceptual and empirical gaps, which urgently need to be filled given the importance of having good models. Model results tell us that details matter:
Table 1 Priority areas for ABR mathematical modelling to inform policy

(1) Explaining population level resistance trends: testing and combining current model structures with diverse multi-level datasets. While there exists a suite of plausible mechanisms that may drive trends in resistance evolution, we currently lack the empirical data to evaluate the relative importance of these mechanisms. To resolve this difficulty, we will have to collect these data and systematically calibrate the suite of models to these data. Doing so will allow us to not only distinguish the underlying mechanism(s), but also to quantify other key parameters such as the strength of selection and competition for a particular bacteria and drug.

(2) Disentangling transmission routes: fitting models to data to generate a standardised modelling framework that shows the pathways of ABR will help to improve intervention targeting as well as to predict future burden.

(3) Translating model predictions to economic outcomes: evaluating the cost-effectiveness of competing ABR control strategies. Although the framework for integrating mathematical model predictions into economic frameworks exists in principle, more work is needed to develop methods specific to antibiotic resistance, such as calculating the shortand long-term costs of antibiotic resistance across priority pathogens and correctly identifying counterfactual scenarios that are contingent on the epidemiology of the pathogen and its setting. Adopting a standardized approach for evaluating the efficiency and optimality of strategies would be invaluable across hospital settings, where arguably the need is greatest.

the strength of selection, the type and strength of fitness costs and the extent of competition between resistant and sensitive strains all change the dynamics of resistance evolution. However, without being able to routinely inform and calibrate these models with comprehensive epidemiologic data, we currently lack confidence in model predictions, most notably at the larger regional and national scale. The potential drivers of resistance evolution that have been supported by or identified using mathematical models are numerous. Empirically testing these hypotheses would allow us to identify the mechanisms that really matter for informing policy.

Hence, whilst modelling has already been useful for developing policy in other areas of infectious disease control and, as such, there exist frameworks for integrating model predictions into an economic evaluation, there is much more to be done before mathematical modelling can robustly underpin ABR control policy. With this in mind, we propose three key goals (Table 1) that, if achieved, will help inform research across the ABR control strategy portfolio.

\section{Abbreviations \\ ABR: Antibiotic resistance; DALYs: Disability-adjusted life years; ECDC EARS- Net: European Centre for Disease Prevention and Control's European Antimicrobial Resistance Surveillance Network; GLASS: World Health Organization's Global Antimicrobial Resistance Surveillance System; JCVI: UK Joint Committee on Vaccination and Immunisation; NITAGs: National Immunization Technical Advisory Groups; QALYs: Quality-adjusted life years}

\section{Acknowledgements}

This discussion paper would not have been possible without the thoughtful presentations from the RESIST workshop speakers and interaction from the attendees. The Centre for Mathematical Modelling of Infectious Diseases and 
the AMR Centre - both at the London School of Hygiene and Tropical Medicine - funded the workshop.

\section{Authors' contributions}

GMK, NGD, LY and KEA wrote the first draft of the manuscript and were responsible for organising the RESIST workshop. All other authors (CC, FC, TD, DRG, REG, MJ, EK, SL, JAL, ML, MJL, ALPM, JVR, MS, DS) were speakers at the RESIST workshop and contributed to the final draft. All authors read and approved the final manuscript.

\section{Funding}

Funding for the RESIST workshop came from the Centre for Mathematical Modelling of Infectious Diseases (CMMID) and the Antimicrobial Resistance Centre at LSHTM. The funding body had no role in the design of the study and collection, analysis, and interpretation of data and in writing the manuscript.

\section{Availability of data and materials}

Not applicable.

\section{Ethics approval and consent to participate}

Not applicable.

\section{Consent for publication}

Not applicable.

\section{Competing interests}

Laith Yakob is a Section Editor of BMC Infectious Diseases. The authors declare that they have no competing interests.

\section{Author details}

${ }^{1}$ Department of Infectious Disease Epidemiology, Faculty of Epidemiology and Population Health, London School of Hygiene and Tropical Medicine (LSHTM), London, UK. ²Department of Mathematics, Simon Fraser University, Burnaby, Canada. ${ }^{3}$ Department of Infection Biology, Faculty of Infectious and Tropical Diseases, LSHTM, London, UK. ${ }^{4}$ Nuffield Department of Medicine, University of Oxford, Oxford, UK. ${ }^{5}$ Faculty of Biology, Medicine and Health, The University of Manchester, Manchester, UK. ${ }^{6}$ Department of Health Services Research and Policy, Faculty of Public Health and Policy, LSHTM, London, UK. ${ }^{7}$ Wellcome Trust, London, UK. ${ }^{8}$ Big Data Institute, Li Ka Shing Centre for Health Information and Discovery, Nuffield Department of Medicine, University of Oxford, Oxford, UK. ${ }^{9}$ Institute for Infection and Immunity, St George's, University of London, Cranmer Terrace, London, UK. ${ }^{10}$ Center for Communicable Disease Dynamics, Department of Epidemiology, Harvard T. H. Chan School of Public Health, Boston, MA, USA. ${ }^{11}$ Department of Global Health and Infection, Brighton and Sussex Medical School, Brighton, UK. ${ }^{12}$ Population Sciences and Pathobiology Department, Royal Veterinary College, London, UK. ${ }^{13}$ Modelling and Economics Unit, National Infection Service, Public Health England, London, UK. ${ }^{14}$ Paediatric Infectious Disease Research Group, St George's University of London, London, UK. ${ }^{15} \mathrm{School}$ of Biosciences, University of Nottingham, Loughborough, UK. ${ }^{16}$ Department of Disease Control, Faculty of Infectious and Tropical Diseases, LSHTM, London, UK. ${ }^{17}$ Centre for Global Health Research, Usher Institute for Population Health Sciences and Informatics, The University of Edinburgh, Edinburgh, UK.

\section{Received: 20 March 2019 Accepted: 11 November 2019} Published online: 29 November 2019

\section{References}

1. Davies J, Davies D. Origins and evolution of antibiotic resistance. Microbiol Mol Biol Rev. 2010;74:417-33

2. Niewiadomska AM, Jayabalasingham B, Seidman JC, Willem L, Grenfell B, Spiro D, et al. Population-level mathematical modeling of antimicrobial resistance: a systematic review. BMC Med. 2019;17:81.

3. Andersson DI, Levin BR. The biological cost of antibiotic resistance. Curr Opin Microbiol. 1999;2:489-93. https://doi.org/10.1016/s1369-5274(99)00005-3.

4. Vogwill T, MacLean RC. The genetic basis of the fitness costs of antimicrobial resistance: a meta-analysis approach. Evol Appl. 2015;8:284-95.

5. Andersson Dl, Hughes D. Antibiotic resistance and its cost: is it possible to reverse resistance? Nat Rev Microbiol. 2010;8:260-71.
6. Blanquart F. Evolutionary epidemiology models to predict the dynamics of antibiotic resistance. Evol Appl. 2019;12:365-83.

7. Negri MC, Lipsitch M, Blázquez J, Levin BR, Baquero F. Concentrationdependent selection of small phenotypic differences in TEM betalactamase-mediated antibiotic resistance. Antimicrob Agents Chemother. 2000;44:2485-91.

8. Wargo AR, Huijben S, de Roode JC, Shepherd J, Read AF. Competitive release and facilitation of drug-resistant parasites after therapeutic chemotherapy in a rodent malaria model. Proc Natl Acad Sci U S A. 2007; 104:19914-9. https://doi.org/10.1073/pnas.0707766104.

9. Lewnard JA, Tähtinen PA, Laine MK, Lindholm L, Jalava J, Huovinen P, et al. Impact of antimicrobial treatment for acute otitis media on carriage dynamics of penicillin-susceptible and penicillin-nonsusceptible Streptococcus pneumoniae. J Infect Dis. 2018;218:1356-66.

10. Davies NG, Flasche S, Jit M, Atkins KE. Within-host dynamics shape antibiotic resistance in commensal bacteria. Nat Ecol Evol. 2019;3:440-9.

11. Colijn C, Cohen T. How competition governs whether moderate or aggressive treatment minimizes antibiotic resistance. Elife. 2015;4. https:// doi.org/10.7554/eLife.10559.

12. Yakob L, Riley TV, Paterson DL, Marquess J, Soares Magalhaes RJ, FuruyaKanamori $\mathrm{L}$, et al. Mechanisms of hypervirulent Clostridium difficile ribotype 027 displacement of endemic strains: an epidemiological model. Sci Rep. 2015;5. https://doi.org/10.1038/srep12666.

13. Davies NG, Flasche S, Jit M, Atkins KE. Competition and diversity determine vaccine impact on antibiotic resistance evolution: bioRxiv preprint. https:// doi.org/10.1101/610188.

14. van Schaik W. The human gut resistome. Philos Trans R Soc Lond B Biol Sci. 2015:370:20140087.

15. Austin DJ, Anderson RM. Studies of antibiotic resistance within the patient, hospitals and the community using simple mathematical models. Philos Trans R Soc Lond B Biol Sci. 1999;354:721-38.

16. Cooper BS, Medley GF, Stone SP, Kibbler CC, Cookson BD, Roberts JA, et al. Methicillin-resistant Staphylococcus aureus in hospitals and the community: stealth dynamics and control catastrophes. Proc Natl Acad Sci U S A. 2004;101:10223-8.

17. Donker T, Henderson KL, Hopkins KL, Dodgson AR, Thomas S, Crook DW, et al. The relative importance of large problems far away versus small problems closer to home: insights into limiting the spread of antimicrobial resistance in England. BMC Med. 2017;15:86.

18. Wei Y, Kypraios T, O'Neill PD, Huang SS, Rifas-Shiman SL, Cooper BS. Evaluating hospital infection control measures for antimicrobial-resistant pathogens using stochastic transmission models: Application to vancomycin-resistant enterococci in intensive care units. Stat Methods Med Res. 2018;27:269-85.

19. Mather AE, Vaughan TG, French NP. Molecular approaches to understanding transmission and source attribution in nontyphoidal salmonella and their application in Africa. Clin Infect Dis. 2015;61(Suppl 4):S259-65.

20. Knight GM, Costelloe C, Deeny SR, Moore LSP, Hopkins S, Johnson AP, et al. Quantifying where human acquisition of antibiotic resistance occurs: a mathematical modelling study. BMC Med. 2018;16:137.

21. MacFadden DR, Fisman DN, Hanage WP, Lipsitch M. The relative impact of community and hospital antibiotic use on the selection of extended-spectrum beta-lactamase-producing Escherichia coli. Clin Infect Dis. 2019;69:182-8.

22. van Bunnik BAD, Woolhouse MEJ. Modelling the impact of curtailing antibiotic usage in food animals on antibiotic resistance in humans. R Soc Open Sci. 2017:4:161067.

23. Smith DL, Harris AD, Johnson JA, Silbergeld EK, Morris JG. Animal antibiotic use has an early but important impact on the emergence of antibiotic resistance in human commensal bacteria. Proc Natl Acad Sci. 2002;99:64349. https://doi.org/10.1073/pnas.082188899.

24. Pires SM, Duarte AS, Hald T. Source attribution and risk assessment of antimicrobial resistance. Microbiol Spectr. 2018;6. https://doi.org/10.1128/ microbiolspec.ARBA-0027-2017.

25. Ludden C, Raven KE, Jamrozy D, Gouliouris T, Blane B, Coll F, et al. One health genomic surveillance of Escherichia coli demonstrates distinct lineages and mobile genetic elements in isolates from humans versus livestock. MBio. 2019;10. https://doi.org/10.1128/mBio.02693-18.

26. Goossens H, Ferech $M$, Vander Stichele R, Elseviers M, ESAC Project Group. Outpatient antibiotic use in Europe and association with resistance: a crossnational database study. Lancet. 2005;365:579-87.

27. ECDC (European Centre for Disease Prevention and Control), EFSA (European Food Safety Authority), EMA (European Medicines Agency). ECDC/EFSA/EMA 
second joint report on the integrated analysis of the consumption of antimicrobial agents and occurrence of antimicrobial resistance in bacteria from humans and food-producing animals-Joint Interagency Antimicrobial Consumption and Resistance Analysis (JIACRA) Report. EFSA J. 2017;15:4872.

28. Colijn C, Cohen T, Fraser C, Hanage W, Goldstein E, Givon-Lavi N, et al. What is the mechanism for persistent coexistence of drug-susceptible and drugresistant strains of Streptococcus pneumoniae? J R Soc Interface. 2010;7:905-19.

29. Lehtinen S, Blanquart F, Croucher NJ, Turner P, Lipsitch M, Fraser C. Evolution of antibiotic resistance is linked to any genetic mechanism affecting bacterial duration of carriage. Proc Natl Acad Sci U S A. 2017;114:1075-80.

30. Lehtinen S, Blanquart F, Lipsitch M, Fraser C, with the Maela Pneumococcal Collaboration. On the evolutionary ecology of multidrug resistance in bacteria. PLoS Pathog. 2019;15:e1007763.

31. Blanquart F, Lehtinen S, Lipsitch M, Fraser C. The evolution of antibiotic resistance in a structured host population. J R Soc Interface. 2018;15: 20180040. https://doi.org/10.1098/rsif.2018.0040.

32. European Centre for Disease Prevention and Control (ECDC). Data from the ECDC Surveillance Atlas - Antimicrobial resistance. https://ecdc.europa.eu/ en/antimicrobial-resistance/surveillance-and-disease-data/data-ecdc. Accessed 1 June 2019.

33. Center for Disease Dynamics, Economics \& Policy (CDDEP). ResistanceMap. https://resistancemap.cddep.org. Accessed 1 June 2019.

34. World Health Organization (WHO). Global Antimicrobial Resistance Surveillance System (GLASS). https://www.who.int/glass/. Accessed 1 June 2019.

35. Cobey S, Baskerville EB, Colijn C, Hanage W, Fraser C, Lipsitch M. Host population structure and treatment frequency maintain balancing selection on drug resistance. J R Soc Interface. 2017;14. https://doi.org/10.1098/rsif.2017.0295.

36. Knight GM, Zimic M, Funk S, Gilman RH, Friedland JS, Grandjean L. The relative fitness of drug-resistant Mycobacterium tuberculosis: a modelling study of household transmission in Peru. J R Soc Interface. 2018;15. https:// doi.org/10.1098/rsif.2018.0025.

37. Whittles LK, White PJ, Didelot X. Estimating the fitness cost and benefit of cefixime resistance in Neisseria gonorrhoeae to inform prescription policy: a modelling study. PLoS Med. 2017;14:e1002416. https://doi.org/10.1371/ journal.pmed.1002416.

38. Maher MC, Alemayehu W, Lakew T, Gaynor BD, Haug S, Cevallos V, et al. The fitness cost of antibiotic resistance in Streptococcus pneumoniae: insight from the field. PLoS One. 2012;7:e29407.

39. Salvatore PP, Becerra MC, Abel zur Wiesch P, Hinkley T, Kaur D, Sloutsky A, et al. Fitness costs of drug resistance mutations in multidrug-resistant Mycobacterium tuberculosis: a household-based case-control study. J Infect Dis. 2016;213:149-55.

40. Gifford DR, Moss E, Craig MacLean R. Environmental variation alters the fitness effects of rifampicin resistance mutations in Pseudomonas aeruginosa. Evolution. 2016;70:725-30. https://doi.org/10.1111/evo.12880.

41. Gifford DR, Krašovec R, Aston E, Belavkin RV, Channon A, Knight CG. Environmental pleiotropy and demographic history direct adaptation under antibiotic selection. Heredity. 2018;121:438-48.

42. Russ D, Kishony R. Additivity of inhibitory effects in multidrug combinations. Nat Microbiol. 2018;3:1339-45.

43. van Kleef E, Robotham JV, Jit M, Deeny SR, Edmunds WJ. Modelling the transmission of healthcare associated infections: a systematic review. BMC Infect Dis. 2013;13:294.

44. Robotham JV, Deeny SR, Fuller C, Hopkins S, Cookson B, Stone S. Costeffectiveness of national mandatory screening of all admissions to English National Health Service hospitals for meticillin-resistant Staphylococcus aureus: a mathematical modelling study. Lancet Infect Dis. 2016;16:348-56.

45. Atkins KE, Lafferty El, Deeny SR, Davies NG, Robotham JV, Jit M. Use of mathematical modelling to assess the impact of vaccines on antibiotic resistance. Lancet Infect Dis. 2018;18:e204-13.

46. Yakob L, Riley TV, Paterson DL, Marquess J, Clements AC. Assessing control bundles for Clostridium difficile: a review and mathematical model. Emerg Microbes Infect. 2014;3:e43.

47. Garnett GP, Cousens S, Hallett TB, Steketee R, Walker N. Mathematical models in the evaluation of health programmes. Lancet. 2011;378:515-25.

48. Joint Committee on Vaccination and Immunisation (JCVI). https://www.gov. uk/government/groups/joint-committee-on-vaccination-and-immunisation. Accessed 1 June 2019.

49. Naylor NR, Atun R, Zhu N, Kulasabanathan K, Silva S, Chatterjee A, et al. Estimating the burden of antimicrobial resistance: a systematic literature review. Antimicrob Resist Infect Control. 2018;7:58.
50. Cassini A, Högberg LD, Plachouras D, Quattrocchi A, Hoxha A, Simonsen GS, et al. Attributable deaths and disability-adjusted life-years caused by infections with antibiotic-resistant bacteria in the EU and the European Economic Area in 2015: a population-level modelling analysis. Lancet Infect Dis. 2019;19:56-66.

51. Chae C, Davies NG, Jit M, Atkins KE. Effect of pediatric Influenza vaccination on antibiotic resistance, England and Wales. Emerg Infect Dis. 2020;26(1) (epub ahead of print)). https://doi.org/10.3201/eid2601.191110.

52. Laxminarayan R, Duse A, Wattal C, Zaidi AKM, Wertheim HFL, Sumpradit N, et al. Antibiotic resistance-the need for global solutions. Lancet Infect Dis. 2013;13:1057-98.

53. National Institute for Health and Care Excellence (NICE). Antimicrobial stewardship: prescribing antibiotics. https://www.nice.org.uk/advice/ktt9/ chapter/evidence-context. Accessed 1 June 2019.

54. Blanquart F, Lehtinen S, Fraser C. An evolutionary model to predict the frequency of antibiotic resistance under seasonal antibiotic use, and an application to Streptococcus pneumoniae. Proc Biol Sci. 2017;284. https://doi. org/10.1098/rspb.2017.0679.

55. Lipsitch M, Bergstrom CT, Levin BR. The epidemiology of antibiotic resistance in hospitals: paradoxes and prescriptions. Proc Natl Acad Sci U S A. 2000;97:1938-43.

56. Ultsch B, Damm O, Beutels P, Bilcke J, Brüggenjürgen B, Gerber-Grote A et al. Methods for health economic evaluation of vaccines and immunization decision frameworks: a consensus framework from a European vaccine economics community. Pharmacoeconomics. 2016;34:227.

57. National Institute for Health and Care Excellence (NICE). Guide to the methods of technology appraisal 2013. https://www.nice.org.uk/process/pmg9/resources/ guide-to-the-methods-of-technology-appraisal-2013-pdf-2007975843781. Accessed 1 June 2019.

58. The Lancet Respiratory Medicine. Antimicrobial resistance-what can we learn from climate change? Lancet Respir Med. 2016:4:845.

59. Review on Antimicrobial Resistance: tackling drug-resistant infections globally. https://amr-review.org/Publications.html. Accessed 1 June 2019.

60. Cravo Oliveira Hashiguchi T, Ait Ouakrim D, Padget M, Cassini A, Cecchini M Resistance proportions for eight priority antibiotic-bacterium combinations in OECD, EU/EEA and G20 countries 2000 to 2030: a modelling study. Euro Surveill. 2019;24. https://doi.org/10.2807/1560-7917.ES.2019.24.20.1800445.

61. de Kraker MEA, Stewardson AJ, Harbarth S. Will 10 million people die a year due to antimicrobial resistance by 2050? PLoS Med. 2016, e1002184;13.

62. Mostofsky E, Lipsitch M, Regev-Yochay G. Is methicillin-resistant Staphylococcus aureus replacing methicillin-susceptible S. aureus? J Antimicrob Chemother. 2011;66:2199-214.

63. Goldstein E, MacFadden DR, Karaca Z, Steiner CA, Viboud C, Lipsitch M. Antimicrobial resistance prevalence, rates of hospitalization with septicemia and rates of mortality with sepsis in adults in different US states. Int J Antimicrob Agents. 2019;54:23-34.

64. Laxminarayan R, Klugman KP. Communicating trends in resistance using a drug resistance index. BMJ Open. 2011;1:e000135.

65. Pouwels KB, Freeman R, Muller-Pebody B, Rooney G, Henderson KL, Robotham JV, et al. Association between use of different antibiotics and trimethoprim resistance: going beyond the obvious crude association. J Antimicrob Chemother. 2018;73:1700-7.

66. Smieszek T, Pouwels KB, Dolk FCK, Smith DRM, Hopkins S, Sharland M, et al. Potential for reducing inappropriate antibiotic prescribing in English primary care. J Antimicrob Chemother. 2018;73:ii36-43.

67. Aldeyab MA, Scott MG, Kearney MP, Alahmadi YM, Magee FA, Conlon G, et al. Impact of an enhanced antibiotic stewardship on reducing methicillinresistant Staphylococcus aureus in primary and secondary healthcare settings. Epidemiol Infect. 2014;142:494-500.

68. Lawes T, Lopez-Lozano J-M, Nebot CA, Macartney G, Subbarao-Sharma R, Dare $C R$, et al. Effects of national antibiotic stewardship and infection control strategies on hospital-associated and community-associated meticillin-resistant Staphylococcus aureus infections across a region of Scotland: a non-linear time-series study. Lancet Infect Dis. 2015;15:1438-49.

69. Lawes T, Lopez-Lozano J-M, Nebot CA, Macartney G, Subbarao-Sharma R, Wares KD, et al. Effect of a national 4C antibiotic stewardship intervention on the clinical and molecular epidemiology of Clostridium difficile infections in a region of Scotland: a non-linear time-series analysis. Lancet Infect Dis. 2017:17:194-206.

70. Timbrook TT, Hurst JM, Bosso JA. Impact of an antimicrobial stewardship program on antimicrobial utilization, bacterial susceptibilities, and financial expenditures at an Academic Medical Center. Hosp Pharm. 2016;51:703-11. 
71. Baur D, Gladstone BP, Burkert F, Carrara E, Foschi F, Döbele S, et al. Effect of antibiotic stewardship on the incidence of infection and colonisation with antibiotic-resistant bacteria and Clostridium difficile infection: a systematic review and meta-analysis. Lancet Infect Dis. 2017;17:990-1001.

72. Pouwels KB, Batra R, Patel A, Edgeworth JD, Robotham JV, Smieszek T. Will co-trimoxazole resistance rates ever go down? Resistance rates remain high despite decades of reduced co-trimoxazole consumption. J Glob Antimicrob Resist. 2017;11:71-4.

73. Arnold SR, Straus SE. Interventions to improve antibiotic prescribing practices in ambulatory care. Cochrane Database Syst Rev. 2005;(4):CD003539.

74. Olesen SW, Barnett ML, MacFadden DR, Brownstein JS, Hernández-Díaz S, Lipsitch M, et al. The distribution of antibiotic use and its association with antibiotic resistance. Elife. 2018;7. https://doi.org/10.7554/eLife.39435.

75. van Duijn PJ, Verbrugghe W, Jorens PG, Spöhr F, Schedler D, Deja M, et al. The effects of antibiotic cycling and mixing on antibiotic resistance in intensive care units: a cluster-randomised crossover trial. Lancet Infect Dis. 2018;18:401-9

76. Lipsitch M, Siber GR. How Can Vaccines Contribute to Solving the Antimicrobial Resistance Problem? MBio. 2016;7. https://doi.org/10.1128/mBio.00428-16.

77. Obolski U, Lourenço J, Thompson C, Thompson R, Gori A, Gupta S. Vaccination can drive an increase in frequencies of antibiotic resistance among nonvaccine serotypes of Streptococcus pneumoniae. Proc Natl Acad Sci U S A. 2018;115:3102-7.

78. Yelin I, Snitser O, Novich G, Katz R, Tal O, Parizade M, et al. Personal clinical history predicts antibiotic resistance of urinary tract infections. Nat Med. 2019;25:1143-52.

79. Tests for rapidly identifying bloodstream bacteria and fungi (LightCycler SeptiFast Test MGRADE, SepsiTest and IRIDICA BAC BSI assay). https://www. nice.org.uk/guidance/dg20. Accessed 1 June 2019

80. Procalcitonin testing for diagnosing and monitoring sepsis (ADVIA Centaur BRAHMS PCT assay, BRAHMS PCT Sensitive Kryptor assay, Elecsys BRAHMS PCT assay, LIAISON BRAHMS PCT assay and VIDAS BRAHMS PCT assay). https://www.nice.org.uk/guidance/dg18. Accessed 1 June 2019.

81. Tuite AR, Gift TL, Chesson HW, Hsu K, Salomon JA, Grad YH. Impact of rapid susceptibility testing and antibiotic selection strategy on the emergence and spread of antibiotic resistance in gonorrhea. J Infect Dis. 2017;216:1141-9.

82. Keenan JD, Bailey RL, West SK, Arzika AM, Hart J, Weaver J, et al. Azithromycin to reduce childhood mortality in Sub-Saharan Africa. N Engl J Med. 2018:378:1583-92.

83. Knight GM, Costelloe C, Murray KA, Robotham JV, Atun R, Holmes AH. Addressing the unknowns of antimicrobial resistance: quantifying and mapping the drivers of burden. Clin Infect Dis. 2018;66:612-6.

84. World Health Organization, Food and Agriculture Organization of the United Nations, World Organisation for Animal Health. Antimicrobial resistance: a manual for developing national action plans, version 1. World Health Organization. https://apps.who.int/iris/handle/10665/204470. Accessed 1 June 2019

85. Munk P, Andersen VD, de Knegt L, Jensen MS, Knudsen BE, Lukjancenko O, et al. A sampling and metagenomic sequencing-based methodology for monitoring antimicrobial resistance in swine herds. J Antimicrob Chemother. 2017;72:385-92.

\section{Publisher's Note}

Springer Nature remains neutral with regard to jurisdictional claims in published maps and institutional affiliations.

Ready to submit your research? Choose BMC and benefit from:

- fast, convenient online submission

- thorough peer review by experienced researchers in your field

- rapid publication on acceptance

- support for research data, including large and complex data types

- gold Open Access which fosters wider collaboration and increased citations

- maximum visibility for your research: over $100 \mathrm{M}$ website views per year

At $\mathrm{BMC}$, research is always in progress.

Learn more biomedcentral.com/submissions 